\title{
Light-cone gauge singularities in the photon propagator and residual gauge transformations
}

\section{Bruno Mutet and Pierre Grangé*}

Laboratoire de Physique Théorique et Astroparticules

Université de Montpellier II, CNRS, IN2P3,

Pl. E. Bataillon, Montpellier, Cedex 05, F-34095, France

E-mail:

pierre.grange@lpta.univ-montp2.fr, bruno.mutet@lpta.univ-montp2.fr

\section{Ernst Werner}

Institut für Theoretische Physik

D-93040, Universität Regensburg - Germany

E-mail: egwerner@gmx.de

\begin{abstract}
The photon propagator is built in the usual light-cone gauge $\left(n^{2}=n \cdot A=\partial \cdot A=0\right)$ within the distributional approach with test functions for the gauge fields. It is shown that test functions provide a sound definition, as distributions, of singular terms in $\frac{1}{(n . k)^{p}}$. The relevance of these extensions is examplified in a test case where an infinite resummation in powers of $\frac{1}{n . k}$ leads to known exact results. The effect of residual gauge degrees of freedom on the presence of singular terms in the most general LC-propagator is discussed in the Dirac-Bergmann algorithm.
\end{abstract}

LIGHT CONE 2008 Relativistic Nuclear and Particle Physics

July 7-11 2008

Mulhouse, France

\footnotetext{
* Speaker.
} 


\section{Introduction}

The light-cone (LC) gauge is one of the most frequently used gauge choices in perturbative QCD calculations and in non-perturbative light-front approaches. In these latter cases, besides the conveniences already known from perturbative studies -built-in transversality of the Green's functions, ghost-free procedure etc...- the LC-gauge turns out to simplify greatly the treatment of constraints inherent to LC dynamics. However difficulties and inconsistencies remain in LCquantization of gauge fields. They have to do with specific spurious singularities appearing in the LC-gauge-field propagator. These questions are most carefully discussed in the essential books of A.Bassetto, G. Nardelli and R. Soldati [1] and of G. Leibbrandt [2], where additional references can be found. More recently A. Das et al. [3] examined the effect of residual gauge fixing on the large distance behaviour of the propagator with Mandelstam-Leibbrandt [ML] prescription for the spurious singularities. In [1] it is emphasized that in order to give a meaning to powers of these spurious singularities the distributional nature of Green's functions has to be taken into account, that is these distributions act on a space of test functions (known as Besov space) suitably vanishing at the singularities. Each vertex of a diagram carries an adiabatic switching test function which ultimately tends to unity at the end of the calculations. Our purpose here is to show that the practical implementation of these ideas is precisely accomplished with the treatment of gauge fields as operator-valued distributions (OPVD) acting on very specific test functions [ 4 - 6]. In the first section OPVD gauge fields are introduced and the photon propagator is derived in the LC-gauge. In the next section the general features of the treatment of singular distributions with partition of unity test functions is recalled and applied to the propagator. The third section presents a test case where an infinite resummation in the LC-singularities gives back the known exact result. This example also brings some light on the Mandelstam-Leibbrandt prescription for the LC-singularity in the general context of the present extension of singular distributions. In the last section residual gauge tansformations and singularities in the photon propagator are discussed from the point of view that the original light cone vector $n$ and its dual $n^{*}-\left(\left(n^{*}\right)^{2}=0, n \cdot n^{*}=1\right)$ - play a completely symmetric role for the implementation of the gauge conditions. Some general comments and perspectives are finally given in the closing section.

\section{Propagator with OPVD gauge fields in the LC-gauge}

An operator-valued distribution defines an operator functional with respect to a $C^{\infty}$ test function $\rho(x)$, with compact support. For the gauge field this operator functional writes

$$
\mathbf{A}_{\mu}(\rho) \equiv<A_{\mu}, \rho>=\int d^{(D)} y A_{\mu}(y) \rho(y) .
$$

The translated functional is a well defined object such that

$$
T_{x} \mathbf{A}_{\mu}(\rho)=<T_{x} A_{\mu}, \rho>=<A_{\mu}, T_{-x} \rho>\equiv \mathbf{A}_{\mu}(x)=\int d^{(D)} y A_{\mu}(y) \rho(y-x) .
$$

Under a gauge transformation of the original $A_{\mu}(y) \mathbf{A}_{\mu}(x)$ get transformed as

$$
\mathbf{A}^{\prime \mu}(x)=\mathbf{A}^{\mu}(x)+\int d^{(D)} y \partial_{y}^{\mu} \Lambda(y) \rho(x-y)=\mathbf{A}^{\mu}(x)+\partial_{x}^{\mu} \int d^{(D)} y \Lambda(y) \rho(x-y)=\mathbf{A}^{\mu}(x)+\partial_{x}^{\mu} \mathbf{\Lambda}(x) .
$$


$\mathbf{A}_{\mu}(x)$ is then taken as the physical field from which the propagator is constructed.

The propagator $\mathbf{G}_{\mu v}(x)$ is formed equivalently ${ }^{1}$ either by the time-ordered product of two $\mathbf{A}_{\mu}$ or by the convolution of the time-ordered product of two $A_{\mu}$ fields with two test functions and the use of their $\mathscr{F}$-transforms $(f=\mathscr{F}(\rho))$

$$
\begin{aligned}
\mathbf{G}_{\mu v}(x) & =\int d^{(D)} z d^{(D)} y^{\prime}<0\left|T\left[A_{\mu}(z+x) A_{v}\left(y^{\prime}\right)\right]\right| 0>\rho(z) \rho\left(y^{\prime}\right), \\
& =\int d^{(D)} z d^{(D)} y^{\prime} G_{\mu v}\left(z+x-y^{\prime}\right) \rho(z) \rho\left(y^{\prime}\right)=\imath \int \frac{d^{(D)} k}{(2 \pi)^{D}} \frac{\exp ^{[-\imath k . x]}}{k^{2}+\imath \varepsilon} D_{\mu v}(k) f^{2}\left[k_{0}^{2}, \vec{k}^{2}\right] .
\end{aligned}
$$

In the "usual" LC gauges with $n^{2}=0, D_{\mu v}(k)=-g_{\mu \nu}+\frac{n_{\mu} k_{v}+n_{v} k_{\mu}}{n \cdot k}-\frac{n_{\mu} n_{v}}{(n \cdot k)^{2}} k^{2}$. In the absence of test functions the common wisdom is to treat the singularity in $\frac{1}{n \cdot k}$ with the ML prescription. It is worthwile to recall that such a prescription is compatible with the path integral formulation [7] but not imposed by it [1]. Different prescriptions for the spurious pole are possible depending on the gauge field boundary conditions. However the definition of higher powers of the spurious singularity is not settled by the ML prescription. Moreover -as observed in [阿- no complete regularisation of the singularity is achieved when $n . k$ and $n^{*} . k \rightarrow 0$ simultaneously and non local ultraviolet divergent terms show up in loop diagrams with the ML prescription. Due to the presence of test functions in $\mathbf{G}_{\mu \nu}$ a different analysis is possible which is in keeping with generic mathematical extension of singular distributions.

\section{Generic extension of singular distributions and application to $\mathbf{G}_{\mu \nu}$}

Let us consider now a singular distribution $T(X) \in \mathbb{S}^{\prime}\left(\mathbb{R}^{d}-\{0\}\right)$. The singular order $k$ of $T(X)$ at the origin of $\left(\mathbb{R}^{d}\right)$ is such that $k=\inf \left\{s: \lim _{\lambda \rightarrow 0} \lambda^{s} T(\lambda X)=0\right\}-d$. We shall call $\widetilde{T}(X)$ the extension to the whole of $\mathbb{S}^{\prime}\left(\mathbb{R}^{d}\right)$ of the original $T(X)$. The construction of $\widetilde{T}(X)$ is achieved with a super regular test function $\left.(\mathrm{SRTF}) \in \mathbb{S}^{(} \mathbb{R}^{d}\right)$ constructed as a partition of unity (PoU). A function is dubbed SRTF if it vanishes with all its derivatives at the boundaries of its domain of definition and a PoU is made of the superposition of functions $u(x-j h)$ suh that for $x \in[j h,(j+1) h] \quad u(x-j h)+u((j+1) h-x)=1[6]$.

For a SRTF $f$ is identical to its Taylor series remainder [9] $R_{0}^{k} f$ of any order since $f^{(\alpha)}(0)=0$ $\forall \alpha \geq 0$.

By construction [6] a PoU can be written as $\quad f(X)=w(X) g(X) \quad$ with $\quad g(X) \leq 1 \quad$ and $w\left(\frac{X}{t}\right)=$ $\theta(t-\tilde{\mu}\|X\|), \tilde{\mu} \leq 1$. We consider now a singular homogeneous distribution $T(X)$ such that $T\left(\frac{X}{t}\right)=$ $t^{(k+d)} T(X)$. The general analysis using Lagrange's formula [5, 6, 8, 9 ] for the Taylor series remainder and presented in former LC-meetings leads to the space(-time) integral

$$
\begin{aligned}
<T(X), w(X) g(X)> & \left.=(-)^{k+1}(k+1) \sum_{|\beta|=k+1} \int d^{d} X \partial_{X}^{\beta}\left[\frac{X^{\beta}}{\beta !} T(X)\right] \int_{\tilde{\mu}\|X\|}^{1} d t \frac{(1-t)^{k}}{t}\right] g(X) \\
& =<\widetilde{T}(X), g(X)>
\end{aligned}
$$

\footnotetext{
${ }^{1}$ due to the specific properties of the test functions (Eq. IV.12 of Ref.[阿)
} 
After the final $t$-integration the extension $\widetilde{T}(X)$ of $T(X)$ is read off from the $X$-integral and writes

$$
\widetilde{T}(X)=(-)^{k}(k+1) \sum_{|\beta|=k+1} \partial_{X}^{\beta}\left[\frac{X^{\beta}}{\beta !} T(X) \log (\tilde{\mu}\|X\|)\right]+\frac{(-)^{k}}{k !} H_{k} \sum_{|\beta|=k} C^{\beta} \delta^{(\beta)}(X)
$$

Here $H_{k}=\sum_{p=1}^{k} \frac{(-1)^{(p+1)}}{p}\left(\begin{array}{l}k \\ p\end{array}\right)=\gamma+\psi(k+1)$ and $C^{\beta}=\int_{(\|X\|=1)} T(X) X^{\beta} d S$.

We envisage now the application of this generic result to $\mathbf{G}_{\mu \nu}$. After integration ${ }^{2}$ over $k^{-}=n^{*} . k$ the following decomposition of $\mathbf{G}_{\mu \nu}$ at $D=4$ is obtained

$$
\begin{aligned}
\mathbf{G}_{\mu v}(x) & =\frac{1}{(2 \pi)^{4}}\left\{-\imath g_{\mu v} I_{0}(X)-\left(n_{v} \partial_{\mu}+n_{\mu} \partial_{v}\right) I_{1}(X)+n_{v} n_{\mu} \partial^{2} I_{2}(X)\right\}, \text { with } \\
I_{p}(X) & =-\imath \pi \int d^{2} k_{\perp} \int_{-\infty}^{\infty} \frac{d(n \cdot k)}{(n \cdot k)^{p+1}} \operatorname{sign}(n \cdot k) \exp [-\imath k \cdot X] f^{2}\left[\frac{1}{2}\left(n \cdot k+\frac{k_{\perp}^{2}}{2 n \cdot k}\right)^{2}\right] .
\end{aligned}
$$

Here $\exp [-\imath k \cdot X]=\exp \left[-\imath\left(n \cdot k X^{-}+\frac{k_{\perp}^{2}}{2 n \cdot k} X^{+}\right)+\imath k_{\perp} \cdot X_{\perp}\right] . \quad f^{2}\left[\frac{1}{2}\left(n \cdot k+\frac{k_{\perp}^{2}}{2 n \cdot k}\right)^{2}\right]$ is a SRTF (of Besov space type [1]) providing extension of distributions in $n . k$ when either $n . k \rightarrow 0$ or $n k \rightarrow \infty$. It gives

$$
\left[\frac{\widetilde{1}}{(n . k)^{p+1}}\right]=\frac{(-)^{p}}{p !} \partial_{n . k}^{p+1} \log [\mu(n . k)]+2 \frac{(-)^{p}}{p !} H_{p} \delta^{(p)}(n . k) .
$$

\section{Relevance of the extension}

A test case is given by the $I_{0}$ piece in the expression for $\mathbf{G}_{\mu v}\left(a=\frac{k_{\perp}^{2}}{2} X^{+}>0, b=X^{-}>0, z=\right.$ $\left.\sqrt{\frac{b}{a}}(n . k)\right)$

$$
I_{f}(a, b)=\int_{0}^{\infty} \frac{d z}{z} \exp \left[\imath \sqrt{a b}\left(z+\frac{1}{z}\right)\right] f\left[\left(z+\frac{1}{z}\right)\right] .
$$

$I_{f}(a, b)$ exists in the limit $f \rightarrow 1$ over the whole integration domain, with the value

$$
I_{f=1}(a, b)=-\pi N_{0}(2 \sqrt{a b})+\imath \pi J_{0}(2 \sqrt{a b}) .
$$

The limit $a b \rightarrow 0$ of $I_{f=1}(a, b)$ (see below) can actually be obtained directly [10] from the integral itself without reference to the above exact result. The strategy is to expand $\exp \left[l \frac{\sqrt{a b}}{z}\right]$ and, at the point where the series does not formally exist, use the extension formula for $\widetilde{\left[\frac{1}{z^{p+1}}\right]}$ and resum. Then

$$
\begin{aligned}
I_{f}(a, b) & =\mathscr{N} \sum_{p=0}^{\infty} \frac{(-\imath \sqrt{a b})^{p}}{(p !)^{2}} \int_{0}^{\infty} \frac{d z}{z} \exp [\imath \sqrt{a b} z]\left[\partial_{z}^{p+1} \log [\mu z]+2 H_{p} \boldsymbol{\delta}^{(p)}(z)\right] \\
& =\mathscr{N} \sum_{p=0}^{\infty} \frac{(-\imath \sqrt{a b})^{p}}{(p !)^{2}} \underset{\varepsilon \rightarrow 0}{\mathscr{L}}\left[\partial_{z}^{p+1} \log [\mu z]+2 H_{p} \boldsymbol{\delta}^{(p)}(z) ; s\right] .
\end{aligned}
$$

\footnotetext{
${ }^{2}$ The test function having no extension to the complex plane, contour techniques cannot be used here; instead the method is that indicated in footnote 1
} 
Here $\mathscr{L}$ is the Laplace-transform, in the sense of distributions, with $s=\varepsilon-\imath \sqrt{a b} \quad \mathfrak{R}(s)>0$

$$
\begin{aligned}
& I_{f}(a, b)=\mathscr{N} \sum_{p=0}^{\infty} \frac{(-1)^{p}(\sqrt{a b})^{2 p}}{(p !)^{2}}\left[\log (\mu)-\log (\sqrt{a b})+\imath \frac{\pi}{2}+\psi(p+1)\right] \\
& \underset{a b \rightarrow 0}{=}-2 \log (\sqrt{a b})-2 \gamma+\imath \pi=\mathscr{N}\left(\log (\mu)-\log (\sqrt{a b})+\imath \frac{\pi}{2}-\gamma\right) .
\end{aligned}
$$

Hence $\mathscr{N}=2, \quad \mu=1$. Regrouping different terms

$$
\begin{aligned}
I_{f}(a, b) & \left.=-2 \sum_{p=0}^{\infty} \frac{(-1)^{p}(\sqrt{a b})^{2 p}}{(p !)^{2}}[\log (\sqrt{a b})+\gamma)\right]+2 \sum_{p=0}^{\infty} \frac{(-1)^{p}(\sqrt{a b})^{2 p}}{(p !)^{2}}(\gamma+\psi(p+1))+\imath \pi J_{0}(2 \sqrt{a b}) \\
& =-\pi N_{0}(2 \sqrt{a b})+\imath \pi J_{0}(2 \sqrt{a b}) .
\end{aligned}
$$

The method gives then a consistent distributional extension not only for the original LC-singularities present in $\mathbf{G}_{\mu \nu}$ but also for any power of them, at variance with the ML prescription.

A more instructive comparison is given when considering the Fourier transforms $G_{M L}$ and $G_{H D}$ of the ML and distributional HD precriptions $\left(p \cdot x=(n \cdot p)\left(n^{*} \cdot x\right)+\left(n^{*} \cdot p\right)(n \cdot x)-p_{\perp} \cdot x_{\perp}\right)$

$$
\begin{aligned}
G_{M L}(x) & =\int \frac{d^{4} p}{(2 \pi)^{4}} \frac{\exp [i p \cdot x]}{n \cdot p+\imath \varepsilon n^{*} \cdot p}=\frac{1}{2 \pi} \frac{1}{n \cdot x-\imath \varepsilon n^{*} \cdot x} \delta^{2}\left(x^{\perp}\right) \\
G_{H D}(x) & =\int \frac{d^{4} p}{(2 \pi)^{4}} \exp [i p \cdot x]\{2 \partial_{n \cdot p}[\log (\mu|n \cdot p|)]+4 \underbrace{H_{0}}_{0} \delta(n \cdot p)\} \\
& =\frac{l}{2 \pi}\left(n^{*} \cdot x\right)\left[4 \pi \gamma \delta\left(n^{*} x\right)+\pi P f\left[\frac{\operatorname{sign}\left(n^{*} \cdot x\right)}{n^{*} \cdot x}\right]\right] \delta(n \cdot x) \delta^{2}\left(x^{\perp}\right)=\frac{l}{2} \operatorname{sign}\left(n^{*} \cdot x\right) \delta(n \cdot x) \delta^{2}\left(x^{\perp}\right)
\end{aligned}
$$

Here $P f$ is the Pseudofunction distribution [11]. Note that both cases correspond to an inversion of $n . \partial$ since $(n . \partial) G_{H D}(x) \equiv \frac{\partial}{\partial\left(n^{*} . x\right)} G_{H D}(x)=(n . \partial) G_{M L}(x)=\imath \delta^{4}(x)$. The HD and ML prescriptions differ in the Pseudofunction part present in the latter, a main characteristic of propagators in the causal approach [Ð]. More comparisons at the level of specific Feynman integrals will be presented elsewhere.

\section{Residual gauge transformations}

On the LC the mutual existence of $n$ and $n^{*}$ implies the following decomposition of the gauge field

$$
\mathbf{A}_{\mu}=\left(n^{*} . \mathbf{A}\right) n_{\mu}+(n . \mathbf{A}) n_{\mu}^{*}+\mathbf{A}_{\mu}^{(T)}=\mathbf{A}_{\mu}^{(L)}+\mathbf{A}_{\mu}^{(T)}
$$

The corresponding projectors onto the transverse and longitudinal directions are given by

$$
\begin{aligned}
& P_{\mu \nu}^{(T)}=\frac{\partial_{\mu} \partial_{v}}{\partial_{\perp}{ }^{2}}+\frac{\left(n^{*} \cdot \partial\right)^{2} n_{\mu} n_{v}}{\partial_{\perp}{ }^{2}}-\frac{n^{*} \cdot \partial\left(n_{\mu} \partial_{\nu}+n_{v} \partial_{\mu}\right)}{\partial_{\perp}{ }^{2}}+\frac{(n \cdot \partial)^{2} n^{*}{ }_{\mu} n^{*}{ }_{v}}{\partial_{\perp}{ }^{2}} \\
& -\frac{n \cdot \partial\left(n^{*}{ }_{\mu} \partial_{v}+n^{*}{ }_{\nu} \partial_{\mu}\right)}{\partial_{\perp}{ }^{2}}-\left(1-\frac{n \cdot \partial n^{*} \cdot \partial}{\partial_{\perp}{ }^{2}}\right)\left(n_{v} n^{*}{ }_{\mu}+n_{\mu} n^{*}{ }_{v}\right)+g_{\mu, v} ; P_{\mu \nu}^{(L)}=g_{\mu, v}-P_{\mu \nu}^{(T)},
\end{aligned}
$$

with the following properties,

$$
\begin{aligned}
& P_{\mu \sigma}^{(T)} P_{v}^{(T) \sigma}=P_{\mu \nu}^{(T)} ; P_{\mu \sigma}^{(L)} \underset{v}{(L) \sigma}=P_{\mu \nu}^{(L)} ; P_{\mu \sigma}^{(L)} P_{v}^{(T) \sigma}=P_{\mu \sigma}^{(T)} P_{v}^{(L) \sigma}=0 . \\
& n^{\mu} P_{\mu \nu}^{(T)}=n^{* \mu} P_{\mu \nu}^{(T)}=\partial^{\mu} P_{\mu \nu}^{(T)}=P_{\mu \nu}^{(T)} n^{v}=P_{\mu \nu}^{(T)} n^{* v}=P_{\mu \nu}^{(T)} \partial^{v}=0 ; \\
& n^{\mu} P_{\mu \nu}^{(L)}=n_{v} ; n^{* \mu} P_{\mu \nu}^{(L)}=n_{v}^{*} ; \partial^{\mu} P_{\mu \nu}^{(L)}=\partial_{v} ; P_{\mu \nu}^{(L)} n^{v}=n_{\mu} ; P_{\mu \nu}^{(L)} n^{* v}=n_{\mu}^{*} ; P_{\mu \nu}^{(L)} \partial^{v}=\partial_{\mu} ;
\end{aligned}
$$


The consequences for $\mathbf{A}_{\mu}^{(L)}=P_{\mu \nu}^{(L)} \mathbf{A}^{v}$ and $\mathbf{A}_{\mu}^{(T)}=P_{\mu \nu}^{(T)} \mathbf{A}^{v}$ are then

$$
\begin{aligned}
(n . \mathbf{A}) & =n^{\mu}\left(P_{\mu \nu}^{(T)}+P_{\mu \nu}^{(L)}\right) \mathbf{A}^{v}=n^{\mu} P_{\mu \nu}^{(L)} \mathbf{A}^{v}=\left(n . \mathbf{A}^{(L)}\right) ; \\
\left(n^{*} . \mathbf{A}\right) & =\left(n^{*} \cdot \mathbf{A}^{(L)}\right) ; \quad(\partial . \mathbf{A})=\left(\partial . \mathbf{A}^{(L)}\right) . \\
\left(n^{*} . \mathbf{A}^{(T)}\right) & =\left(n \cdot \mathbf{A}^{(T)}\right)=\left(\partial . \mathbf{A}^{(T)}\right)=0 .
\end{aligned}
$$

The knowlege of $(\partial . \mathbf{A}),(n . \mathbf{A})$ or $\left(n^{*} . \mathbf{A}\right)$ determines $\mathbf{A}^{(L)}$; $\mathbf{A}^{(T)}$ is gauge invariant: $\mathbf{A}_{\mu}^{\prime(T)}=\mathbf{A}_{\mu}^{(T)}+\underbrace{P_{\mu \nu}^{(T)} \partial^{v}}_{0} \mathbf{\Lambda}=\mathbf{A}_{\mu}^{(T)}$;

$\mathbf{A}^{(L)}$ is not: $\mathbf{A}_{\mu}^{(L)}=\mathbf{A}_{\mu}^{(L)}+P_{\mu \nu}^{(L)} \partial^{v} \mathbf{\Lambda}=\mathbf{A}_{\mu}^{(L)}+\partial_{\mu} \mathbf{\Lambda}$

LC-gauges are such that $(n . \mathbf{A})=0, \quad(\partial . \mathbf{A})=0$ (or same with $\left.n^{*}\right)$.

$$
\begin{aligned}
& (n . \mathbf{A})=0 \rightarrow(n . \partial) \mathbf{\Lambda}=0 \rightarrow \mathbf{\Lambda} \equiv \mathbf{\Lambda}\left(n . x, x_{\perp}\right), \\
& (\partial \mathbf{A})=0 \rightarrow \partial^{2} \mathbf{\Lambda}=\left[2(n . \partial)\left(n^{*} . \partial\right)-\partial_{\perp}^{2}\right] \mathbf{\Lambda}=0 \rightarrow \mathbf{\Lambda}\left(n . x, x_{\perp}\right)=\mathbf{\Lambda}_{1}(n \cdot x)+\mathbf{\Lambda}_{2}^{\perp}(n . x) \cdot x_{\perp}
\end{aligned}
$$

But the gauge transform concerns only $\mathbf{A}^{(L)} \rightarrow \mathbf{\Lambda}_{2}^{\perp}=0$. Imposing in addition $\left(n^{*}\right.$.A $)=0$ eliminates the residual $n . x$ dependence of $\mathbf{\Lambda}$; however is it compatible with the constrained quantization of the gauge field usually performed within the Dirac-Bergmann (DB) algorithm [12]? We first note that $\mathbf{A}_{\mu}$ has a zero mode (P)-sector and a complement, the particle (Q)-sector, where $\mathbf{G}_{\mu \nu}$ acts and where $\left(n . \mathbf{A}^{(T)}\right)=\left(n^{*} \cdot \mathbf{A}^{(T)}\right)=\left(\partial . \mathbf{A}^{(T)}\right)=0$ by construction: the implementation of all gauge conditions (including $\left(n^{*} . \mathbf{A}\right)=0$ ) takes place in the P-sector.

In the DB algorithm the dynamics of the system is finally fixed by the choice of two subsidiary gauge conditions. Any gauge choice should exhaust gauge arbitrariness, that is no more dynamics of redundant degrees of freedom should be present. Already two LC-gauge conditions are imposed $-n . A=0, \partial . A=0$-, but $n . x$ remains as a residual gauge degree of freedom: there is apparently no room for one additional gauge condition. However there is a possible way out since $n \cdot A=0, \partial . A=0 \rightarrow(n \cdot A+\partial . A)^{2}=0 \rightarrow(n \cdot A)^{2}+(\partial . A)^{2}=-2(n . A)(\partial . A)$. Hence the separate Lagrangian enforcement of the two initial gauge conditions is identical to the single enforcement of $(n . A)(\partial . A)=0$, as checked for the classical propagator by Suzuki and Sales [13]. Thus there is some room for an extra gauge condition eliminating the left over redundant degrees of freedom. On LC the decomposition $\mathbf{A}_{\mu}=\left(n^{*} . \mathbf{A}\right) n_{\mu}+(n . \mathbf{A}) n_{\mu}^{*}+\mathbf{A}_{\mu}^{(T)}$ leads to

$$
\begin{gathered}
D_{\mu v}(k)=-g_{\mu, v}-\frac{n_{\mu}^{*} n_{v}^{*}(n \cdot k)^{2}}{k_{\perp}^{2}}+\frac{\left(k_{v} n_{\mu}^{*}+k_{\mu} n_{v}^{*}\right)(n \cdot k)}{k_{\perp}^{2}}-\frac{\left(n^{*} \cdot k\right)^{2} n_{\mu} n_{v}}{k_{\perp}^{2}} \\
+\frac{\left(n^{*} \cdot k\right)\left(k_{v} n_{\mu}+k_{\mu} n_{v}\right)}{k_{\perp}^{2}}+\left(1-\frac{n \cdot k n^{*} \cdot k}{k_{\perp}^{2}}\right)\left(n_{v} n_{\mu}^{*}+n_{\mu} n_{v}^{*}\right)-\frac{k_{\mu} k_{v}}{k_{\perp}^{2}}
\end{gathered}
$$

with the properties of triple transversality and usual closure $D_{\mu \lambda} D_{v}^{\lambda}=-D_{\mu \nu}$

\section{Concluding remarks}

As conjectured long ago by Bassetto et al.[1] the treatment of gauge fields as OPVD with PoU test functions leads to the distributional extensions of the "usual" LC-divergences. These 
extensions are based on a generic mathematical analysis of singular distributions whose principles are not tied up to some a-priori Wick-rotation specificities requested for propagators. It appears that on the light-cone it is necessary to consider the null vector $n$ and its dual $n^{*}$ on the same footing. A specific form of the polarisation tensor $D_{\mu v}(k)$ results from the ensuing transverse and longitidunal projectors. The presence of residual LC-divergences is shown to be connected with an incomplete elimination of redundant degrees of freedom in the Dirac-Bergmann algorithm. After proper distributional extensions the remnant of the test function will provide the necessary regulation in the remaining integrals in the transverse directions. Following [1, 2] and the comments of [3] the general investigation of Feynman integrals within the test function approach is of primary interest and presently under way.

\section{References}

[1] A. Bassetto, G. Nardelli, R. Soldati, Yang-Mills Theories in Algebric Non-Covariant Gauges, World Scientific, Singapore, 1991.

[2] G. Leibbrandt Quantization of Yang-Mills and Chern-Simons Theory in Axial-type Gauges, World Scientific, Singapore, 1994.

[3] A. Das, J. Frenkel, S. Perez, Phys. Rev D70 (125001) 2004 [hep-th/0409081］; A. Das, J. Frenkel, Phys. Rev D71 (087701) 2005 [hep-th/0412071 ]

[4] A. Aste, Finite field theory and causality, $\mathrm{OS}$ (LC2008)

[5] P. Grange, E. Werner, Nucl. Phys.(Proc. Suppl.) B161 (75) 2006 [math-ph/0510052 ].

[6] P. Grange, E. Werner, math-ph/0612011

[7] J. Przeszowski, The Mandelstam-Leibbrandt prescription, CoS (LC2008)

[8] J.M. Gracia-Bondia, Math. Phys. Anal. Geom. 6 (59) 2003 [hep-th/0202023 ]; J.M. Gracia-Bondia and S. Lazzarini, J. Math. Phys. 44 (3863) 2003 [hep-th/0212156 ].

[9] L.S. Gradshteyn,I.M. Ryzhik, Table of Integrals,Series, and Products, Fith edition, Alan Jeffrey, Editor, Academic Press, London, 1994.

[10] R. Estrada, R.P. Kanwall, A Distributional Approach to Asymptotics: Theory and Applications, Birkhaüser, Boston, 2002.

[11] L. Schwartz, Théorie des Distributions, Hermann, Paris, 1963.

[12] K. Sundermayer, Constrained Dynamics, Lectures Notes in Physics 1691982.

[13] A.T. Suzuki, J.H.O. Sales, Nucl.Phs. A275 (139) 2003 [nucl-th/0303016, 0304065 